\title{
ASPECTOS ECOLÓGICOS DA TERMITOFAUNA DE UM REMANESCENTE DE CAATINGA DA REGIÃO NEOTROPICAL, BRASIL
}

\author{
M. P. S. SOUZA , P. A. G. SOUSA \\ Instituto Federal de Educação Ciência e Tecnologia do Rio Grande do Norte, Campus Macau \\ michaelpratini@hotmail.com*
}

Submetido 31/10/2018 - Aceito 07/11/2018

DOI: $10.15628 /$ holos.2018.6489

\section{RESUMO}

A infraordem Isoptera, popularmente conhecida como cupins, compõe-se dos decompositores dominantes e os mais importantes na decomposição da necromassa vegetal e ciclagem de nutrientes. Todavia, poucos estudos sobre a composição e caracterização das suas taxocenoses no bioma da Caatinga foram realizados, em particular no Estado do Rio Grande do Norte. Este trabalho teve como objetivo investigar e descrever a composição, diversidade e riqueza da fauna de térmitas na Floresta Nacional (Flona) Açú, estado do Rio Grande do Norte, Brasil, além de estipular a densidade de ninhos de térmitas por hectare. Para o registro e coleta dos térmitas foi seguido um protocolo padronizado entre abril e maio de 2017, as espécies coletadas foram catalogadas e agrupadas de acordo com a guilda
\end{abstract}

alimentar, hábitos de nidificação, bem como foi estipulado a densidade de ninhos de térmitas/ha. Foram registradas 7 espécies e 12 encontros. A família Termitidae, foi considerada dominante tanto em número de espécies, quanto em número de encontros. A porcentagem de espécies humívoras e xilófagas foram equivalentes, embora os consumidores de madeira tenham sido dominantes em número de encontros. Quanto aos hábitos de nidificação, os valores mais expressivos foram para espécies que constroem seus ninhos no interior de troncos, ou vivem nestes. A densidade, relativamente alta, de ninhos/ha, 131,86 ninhos/ha, estimada para Flona de Açú, revela uma forte presença destes insetos nesta área de Caatinga.

PALAVRAS-CHAVE: Diversidade de Térmitas, Ecologia da Caatinga, Entomologia.

\section{TITULO EM INGLÊS ECOLOGICAL ASPECTS OF THE TERMINE FAUNA A REMANESCENT OF CAATINGA OF THE NEOTROPICAL REGION, BRAZIL}

\begin{abstract}
The Isoptera infraorder, popularly known as termites, is composed of the dominant decomposers and the most important in the decomposition of vegetable necromass and nutrient cycling. However, few studies about composition and characterization of their taxocenoses in the Caatinga biome were carried out, in particular in the State of Rio Grande do Norte. Therefore, the aim of this work was to investigate and describe the composition, diversity and richness of the termite fauna in the National Forest (Flona) Açú, State of Rio Grande do Norte, Brazil, in addition to determining the density of termite nests per hectare. For the registration and collection of termites, a standardized protocol was followed, in the period between April and May 2017, the
\end{abstract}

collected species were cataloged and grouped according to the food guild, nesting habits, as well as the density of termite nests/ha. At end, 7 species and 12 encounters were recorded. The Termitidae family was considered dominant in both number of species and number of encounters. The percentage of humivorous and xylophaga species were equivalent, although wood consumers were dominant in number of encounters. Regarding nesting habits, the most expressive values were for species that build their nests inside or live in logs. The density, relatively high of nests/ha, 131.86 nests/ha estimated for Flona of Açu, shows a strong presence of these insects Caatinga this área.

KEYWORDS: Termite diversity, Caatinga ecology, Entomology. 


\section{INTRODUÇÃO}

\subsection{Domínio morfoclimático da Caatinga}

Dentre os domínios morfoclimáticos brasileiros, o da Caatinga ocupa uma área aproximada de $734.478 \mathrm{~km}^{2}$ (cerca de $11 \%$ do território nacional e $54 \%$ da região nordeste), com todos os seus limites inteiramente restritos ao território do país (Ab'saber, 1974; Vasconcellos et al., 2007), cobrindo além de uma vasta área da região Nordeste, o Norte do estado de Minas Gerais (Prado, 2003). Em relação a sua fitofisionomia, há presença de cactáceas, bromeliáceas, caducidade das folhas e baixa produção de fitomassa (Costa et al., 2007). Esse bioma representa um dos maiores exemplos de ambiente semiárido Neotropical, com elevados índices de evapotranspiração potencial e baixa precipitação, concentrada de três a cinco meses (Sampaio, 2010). Além de altos níveis de radiação solar e de temperatura média anual, baixa nebulosidade e assim como baixas taxas de umidade relativa do ar (Prado, 2003).

Além de ser caracterizada com uma grande taxa de biodiversidade, quando comparada aos outros ecossistemas semiáridos, esta região ainda apresenta um elevado número de espécies endêmicas, sejam vegetais ou animais (Castelletti et al., 2003). Entretanto, a Caatinga se constitui como o bioma brasileiro com o menor status de conservação e com significativas perturbações antrópicas, que chegam a patamares superiores à $50 \%$. O que traduz essa área como fonte de problemáticas relacionadas a reflexos, importância e diminuição da biodiversidade (Maciel, 2010), em especial neste trabalho, aos artrópodes de solo.

\subsection{Taxocenose dos térmitas}

Esse documento já está configurado com as normas pré-estabelecidas pela editor da Revista Holos e, para segui-las, basta substituir os textos de descrição pelo conteúdo do artigo. Caso não seja possível proceder dessa forma, as normas de submissão serão descritas a seguir nos demais itens. A infraordem Isoptera, popularmente conhecida como cupins, possui aproximadamente 3000 espécies descritas, organizadas em 12 famílias (Krishna et al., 2013), das quais quatro são encontradas no Brasil: Kalotermitidae, Rhinotermitidae, Serritermitidae e Termitidae (Santos \& Gomes, 2012). São indivíduos eussociais, compostos por quatro grupos morfológica e funcionalmente distintos: reprodutores e dispersores, operários, soldados e a matéria inanimada representada pelo ninho (Howard \& Thorne, 2011). Estes organismos podem se alimentar de vários tecidos vegetais em diversos estados de decomposição, sendo humívoros quando alimentam-se principalmente de húmus -, e xilófagos - quando alimentam-se diretamente de material vegetal ou uma combinação destes substratos (Lima \& Costa-Leonardo, 2007).

Nos ecossistemas tropicais, esses insetos são considerados os decompositores dominantes e os mais importantes na decomposição da necromassa vegetal e ciclagem de nutrientes (Lo \& Eggleton, 2011), graças a habilidade que possuem de modificar a estrutura do hábitat, através da construção de túneis e galerias que contribuem para aeração, estratificação vertical e descompactação (Bignell \& Eggleton, 2000). Consequentemente, aumentam a retenção 
de água no solo (Ferreira et al., 2011), contrabalanceando o empobrecimento superficial edáfico, imposto pela lixiviação e erosão mecânica (Lima et al., 2017). Esse grupo se constitui ainda como um bioindicador potencial (Holt \& Conventry, 1990), devido a fatores como ampla distribuição geográfica, abundância, hábitos específicos de forrageamento (Eggleton, 2011) e curto tempo de resposta a alterações antrópicas (Vasconcellos et al., 2010). Além da sua importância econômica, no que diz respeito às espécies pragas de agricultura e do ambiente urbano (Rouland-Lefèvre, 2011).

A biodiversidade deste clado no bioma da Caatinga ainda é pouco estudada, sobretudo quanto a composição, riqueza de espécies, grupos alimentares, hábitos de nidificação, abundância e biomassa das populações (Moura et al., 2006), principalmente ao que diz respeito ao estado do Rio Grande do Norte. Aos trabalhos relacionados a este bioma podem ser enumerados Mélo e Bandeira (2007), Menezes (et al., 2008), Bezerra (et al., 2009), Vasconcellos (et al., 2010), Alves (et al., 2011), Barca (2011), Bezerra-Gusmão (et al., 2011), Lustosa Junior (2012), Santos e Gomes (2012), Souza (2012), Bezerra-Gusmão (et al., 2013), Couto (et al., 2015) e Lima (et al., 2017). Com destaque aos trabalhos realizados no Estado do Rio Grande do Norte por Alves e colaboradores (2011) que apresentam os diversos aspectos ecológicos da taxocenose de térmitas na ESEC - Seridó e Souza (2012) que reflete acerca da importância desses insetos na dieta alimentar de lagartos.

Destarte, devido à evidente carência de estudos ecológicos sobre térmitas, em particular no Estado do Rio Grande do Norte, este trabalho teve como objetivo investigar e descrever a composição, diversidade e riqueza da fauna de térmitas na Floresta Nacional (Flona) Açú, estado do Rio Grande do Norte, Brasil, correlacionando com seus hábitos forrageadores e de nidificação, além de estipular a densidade de ninhos de térmitas por hectare da Flona de Açú.

\section{METODOLOGIA}

\section{1 Área de estudo}

A Floresta Nacional (Flona) de Açú é uma unidade de conservação federal localizada a 214 km da capital do Estado do Rio Grande do Norte, abrangendo uma área correspondente a 528,25 ha e situada no município de Assú, Rio Grande do Norte (Figura 1). Apresenta como coordenadas geográficas centrais $05^{\circ} 34^{\prime} 20^{\prime \prime} \mathrm{S}$ e $36^{\circ} 54^{\prime} 33^{\prime \prime} \mathrm{W}$ e, caracteriza-se como um remanescente de floresta de Caatinga (Amorim et al., 2016).

O clima da região é caracteristicamente seco, com uma estação chuvosa que se concentra entre os meses de janeiro a abril, com uma média pluviométrica anual de 585,8 mm, oscilando entre $1,4 \mathrm{~mm}$ à $1.627,1 \mathrm{~mm}$, umidade relativa do ar em torno de $70 \%$ e temperatura média anual de $28,1^{\circ} \mathrm{C}$; o relevo local é predominante suave ondulado e ondulado (PMA, 2015). 


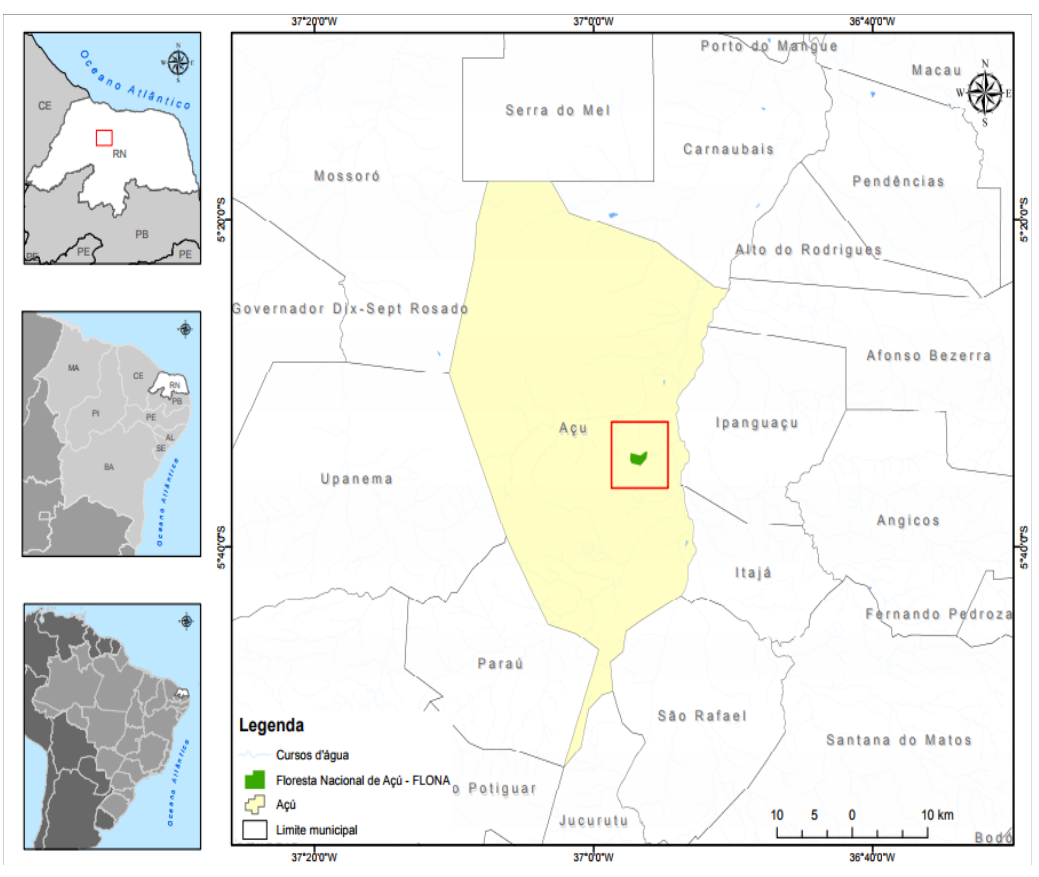

Figura 1. Localização da Floresta Nacional de Açú (Flona Açú) - em verde -, Assú, Rio Grande do Norte, Brasil.

\subsection{Técnicas de coleta}

Para o registro e coleta dos térmitas foram delineados dois transectos de $65 \mathrm{~m} \times 2 \mathrm{~m}$. Em cada transecto foram demarcados pontos de coleta de $5 \times 2 \mathrm{~m}$, equidistantes 10 metros um do outro, totalizando 10 parcelas, com uma área total de $910 \mathrm{~m}^{2}$ (Jones \& Eggleton, 2000). Foram realizadas 5 incursões à campo (todas na estação chuvosa), totalizando 4 dias de coletas. Ressalta-se que todas as idas ao sítio de coleta ocorreram no período diurno e nos meses de abril a maio de 2017.

As coletas ocorreram de forma ativa e direta em troncos ou raízes em decomposição, em galerias, ninhos arborícolas, epígeos e na copa de árvores, procurando desse modo por microhábitats que se encontravam entre $15 \mathrm{~cm}$ de profundidade e $2 \mathrm{~m}$ acima do nível do solo. Prioritariamente, buscou-se indivíduos das castas dos soldados, visto que estes facilitam a identificação (Jones \& Eggleton, 2000). Os espécimes coletados foram acondicionados em recipientes de vidro com álcool $80 \%$ e etiquetados com dados necessários à pesquisa como data, hora, local, pontos de coleta e número do transecto (Jones \& Eggleton, 2000). Quanto a identificação seguiu-se a chave dicotômica proposta por Constantino (1999) e Krishna e colaboradores (2013).

\subsection{Classificação em guildas alimentares}

A partir da observação in situ e revisão da literatura para cada espécie encontrada, os espécimes registrados foram agrupados de acordo com a guilda alimentar e hábitos de nidificação, proposto por diversos autores (Bignell \& Eggleton, 2000; Lima \& Costa-Leonardo, 2007; Vasconcellos et al., 2010; Eggleton, 2011; Souza et al., 2012; Viana Junior et al., 2014), 
sendo classificados em Grupo 1: Consumidores de madeira; Grupo 2: Consumidores de húmus; Grupo 3: Consumidores de madeira/húmus; Grupo 4: Consumidores de serrapilheira.

\subsection{Categorização e densidade dos ninhos}

Neste ponto analisou-se os hábitos de nidificação, observados no momento da coleta, bem como a partir de uma revisão da literatura para cada espécie encontrada, sendo categorizado em 1) ninhos subterrâneos; 2) ninhos epígeos; 3) ninhos arborícolas; 4) ninhos no interior de madeiras (Bignell, 2011). Quanto à densidade de ninhos, demarcou-se a parcela total, a partir do número de encontros estipulou-se a densidade de ninhos para área em hectare. Embora, o procedimento habitual, não se constitua de estipulação, mas da contagem numérica dos ninhos em uma área de um hectare (Alves, et al., 2011).

\subsection{Análises estatísticas}

O esforço amostral foi aferido, com base no número de horas totais, multiplicado pelo número de observadores em campo (horas.homem). A partir dos dados obtidos foi calculado uma curva média da acumulação das espécies/área (curva do coletor), com base no método Mao Tau (Sobs) que possui um intervalo de $95 \%$ de confiança, a fim de verificar se o esforço amostral do trabalho executado foi suficiente para analisar os dados da riqueza total da termitofauna.

Para estimar se a riqueza de espécies obtidas correspondia à prevista para a área, foram utilizados os estimadores não paramétricos de primeira e segunda ordem Jackknife, e Chao de segunda ordem, todos com uma aleatorização de 1000 vezes, com base nos dados de presença/ausência para os dois transectos. Em todos os procedimentos descritos, cada unidade amostral correspondeu à 1 hora de trabalho em campo. Ao passo que os cálculos foram efetuados utilizando-se o software Palaeontological Statistics (Hammer, 2017) e a partir dos resultados obtidos, foram elaborados gráficos, para melhor representatividade, com auxílio do software Microsoft Office Excel 2013.

O número de encontros e a riqueza observada foram utilizadas para análise da abundância da área de estudo, isto posto para diversidade das espécies, estimou-se os resultados com base no índice de diversidade de Shannon-Wiener ( $\left.H^{\prime}\right)$ (Magurran, 2011), que se baseia na abundância relativa das espécies; para este cálculo foi utilizado o programa Palaeontological Statistics. O valor obtido foi comparado com a literatura publicada.

\section{RESULTADOS}

\subsection{Composição, riqueza e diversidade}

No total, foram encontrados 11 espécimes de térmitas na Floresta Nacional (Flona) de Açú, pertencentes a sete espécies, distribuídos em duas famílias e quatro subfamílias. A curva de acumulação de espécies (curva do coletor) obteve uma configuração curva, não assintótica, mesmo após 16 horas de esforço amostral em campo (30 horas.homem), demonstrando, virtualmente, que após contínuas coletas, e mais esforço amostral, mais espécies poderiam ser relatadas (Figura 2a). A curva de rarefação para as espécies observadas (Sobs) apresentou uma 
conformação semelhante e, porém, não longe de obter uma assíntota, expressou que a riqueza de espécies estimadas para a área era 7, variando entre 5,92 a 8,08 (Figura 2b).

O estimador não-paramétrico Jackknife de primeira ordem (Jackk1) previu a ocorrência de 7,4 com desvio padrão de 2,15 (min. 5,25 e max. 9,55) espécies de térmitas para a Flona de Açú, enquanto que o Jackknife de segunda ordem (Jackk2) 7,84 espécies com desvio padrão de 3,56 (min. 4,28 e max. 11,4. Para o Chao 2 foi previsto 6,56 (min. 4,07 e max. 9,05). Com isso, o intervalo de confiança previsto pelo Sobs está incluído nos desvios padrões encontrados para todos os estimadores utilizados, assim como pôde ser observado na figura 3. Por último, o índice de diversidade Shannon-Wienner obtido foi $H^{\prime}=1,86$ (min. 1,23 e max. 1,9).

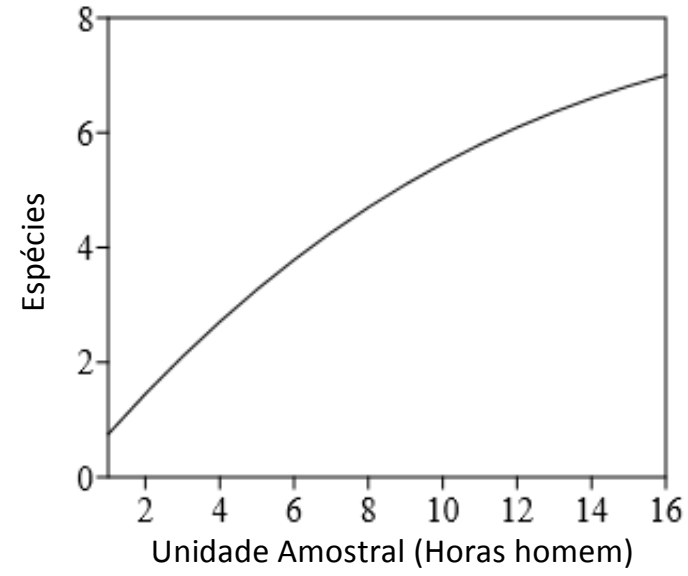

a)

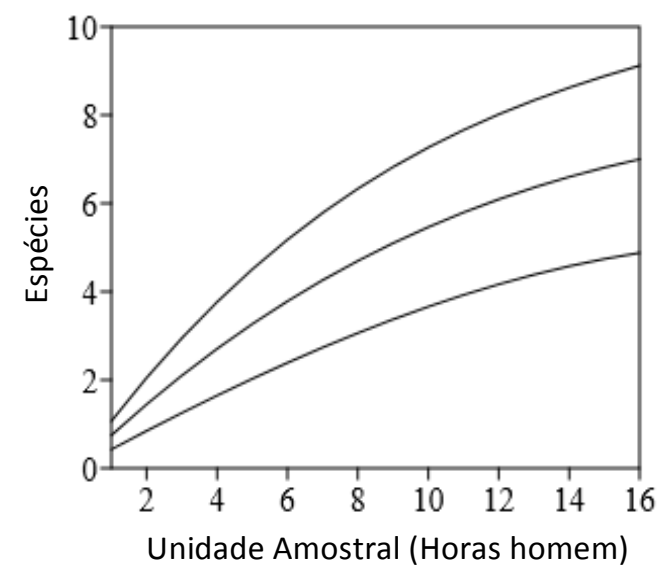

b)

Figura 2. a) Curva de acumulação das espécies de térmitas. b) Curva de rarefação Sobs ( $95 \%$ de Confiança). Flona de Açú, Assú, Rio Grande do Norte, Brasil, no período de abril a maio de 2017.

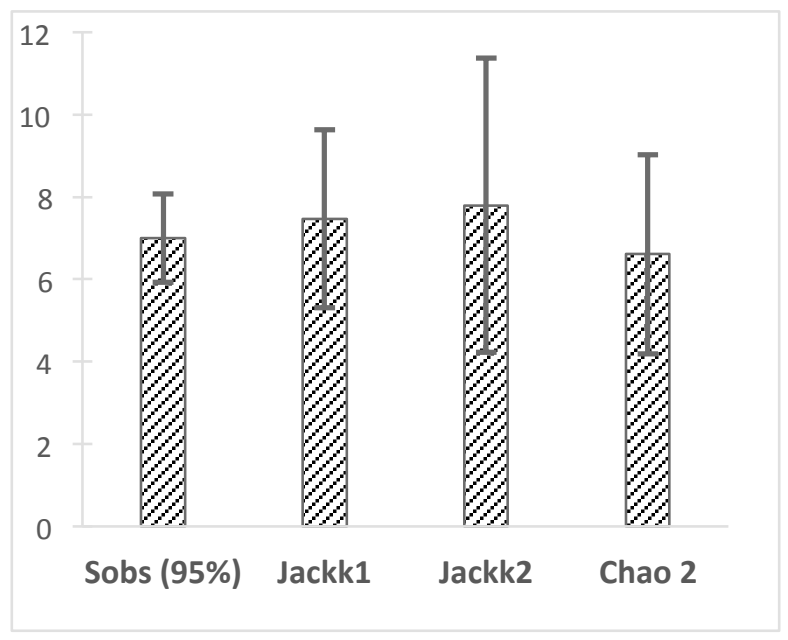

Figura 3. Comparação entre os desvios padrões gerados pelo Sobs e os estimadores Jackknife 1, Jackknife 2 e Chao 2. Flona de Açú, Assú, Rio Grande do Norte, Brasil, no período de abril e maio de 2017.

\subsection{Categorização das espécies encontradas e densidade de ninhos}

Os térmitas registrados foram classificados quanto ao número de encontros, hábitos de nidificação e grupo alimentar (Tabela 1). 
Tabela 1. Número de encontros, hábitos de nidificação e grupo alimentar das espécies de térmitas coletados na Flona de Açú, Assú, Rio Grande do Norte, Brasil, no período de abril a maio de 2017.

\begin{tabular}{|c|c|c|c|}
\hline Espécies & Número de Encontros & Hábitos de Nidificação & Grupo Alimentar \\
\hline \multirow{2}{*}{\multicolumn{4}{|c|}{$\begin{array}{l}\text { Termitidae } \\
\text { Apicotermitinae }\end{array}$}} \\
\hline & & & \\
\hline Anoplotermes sp1. & 1 & Madeira & Humívoro \\
\hline Anoplotermes sp2. & 1 & Madeira & Humívoro \\
\hline Nasutitermitinae & & & \\
\hline Nasutitermes sp. & 1 & Arborícola & Xilófago \\
\hline $\begin{array}{l}\text { Termitinae } \\
\text { Amitermes sp. }\end{array}$ & 3 & Subterrâneo & $\begin{array}{l}\text { Xilófago/Humívora e } \\
\text { Serrapilheira }\end{array}$ \\
\hline Microcerotermes sp. & 2 & Arborícola & Xilófago \\
\hline Termes sp. & 2 & Madeira & Humívoro \\
\hline Rhinotermitidae & & & \\
\hline $\begin{array}{l}\text { Heterotermitinae } \\
\text { Heterotermes } s p .\end{array}$ & 2 & Subterrâneo & Xilófago \\
\hline Total de Espécies & 7 & & \\
\hline Total de Encontros & 12 & & \\
\hline
\end{tabular}

A família Termitidae foi considerada a dominante tanto em número de espécies $(85,71 \%$, $N=7)$, quanto em número de encontros $(83,4 \%, N=12)$. A subfamília Termitinae, incluída na família anteriormente citada, também foi a mais representativa, tanto em número de espécies $(42,85 \%, N=7)$, quanto em número de encontros $(58,4 \%, N=12)$. Baixas representações foram vistas para a subfamília Nasutermitinae (Termitidae), tanto em número de espécies $(14,9 \%, \mathrm{~N}=$ $7)$, quanto em número de encontros $(14,6 \%, N=12)$. A espécie com maior número de encontros foi Amitermes sp. com uma porcentagem expressiva de $25 \%(N=12)$, já Nasutitermes sp. (Termitidae: Nasutitermitinae), Anoplotermes sp1. e Anoplotermes sp2. (Termitidae: Apicotermitinae), foram registradas em apenas um encontro. A segunda família, Rhinotermitidae, obteve uma amostragem de $14,29 \%(N=7)$ das espécies coletadas, sendo o seu único representante pertencente a subfamília Heterotermitinae.

Em relação aos hábitos de nidificação, foram encontradas espécies arborícolas e subterrâneas, em porcentagens equivalentes $(28,57 \%, N=7)$, mas inferior para as espécies que constroem seus ninhos no interior de troncos, ou vivem nestes, que apresentou um valor mais expressivo $(42,85 \%, N=7)$. Em relação ao número de encontros, $25 \%(N=12)$ das espécies coletadas se enquadram no hábito de nidificação arborícola, 33\% ( $N=12)$ nidificam em madeira e $41 \%(\mathrm{~N}=12)$ dos encontros nidificam no solo.

Quanto aos grupos alimentares, 42,85\% ( $N=7)$ das espécies são humívoras, sendo as três espécies, Amitermes sp., Anoplotermes sp1. e Anoplotermes sp2., e curiosamente uma porcentagem equivalente para térmitas de dieta xilófaga, 42,85\% ( $N=7)$, sendo elas Microcerotermes sp., Nasutitermes sp. e Heterotermes sp.. Por último, 14,3\% ( $N=7)$, com uma dieta bem abrangente, Xilófaga, Humívora ou serapilheira, apenas Termes sp.. Em relação ao número de encontros $41 \%(\mathrm{~N}=12)$ foram para espécies xilófagas, 33\% ( $\mathrm{N}=12$ ) para espécies 
humívoras e $25 \%(\mathrm{~N}=12)$ dos encontros para espécies pertencentes a guilda alimentar variada Xilófago/Humívora e Serrapilheira.

No que diz respeito à densidade de ninhos, foi verificado a densidade de 12 ninhos/0,091ha, proporcionalmente 131,86 ninhos/ha. Não obstante, mais três ninhos arborícolas foram avistados, embora sem que houvesse térmitas em seu interior, o que eleva a densidade para 15 ninhos/0,091ha, proporcionalmente 164,83 ninhos/ha.

\section{DISCUSSÃO}

\subsection{Composição, riqueza e diversidade}

O esforço amostral está inteiramente relacionado com a riqueza de espécies que pode ser encontrada em uma dada região (Ugland et al., 2003). O que no trabalho realizado na Flona de Açu pode estar ligado ao fato de que o esforço amostral foi menor do que os executados pelos autores Mélo \& Bandeira, 2007, Menezes (et al., 2008), Bezerra (et al., 2009), Vasconcellos (et al., 2010), Alves (et al., 2011), Barca (2011), Bezerra-Gusmão (et al., 2011) Lustosa Junior (2012), Santos \& Gomes (2012), Souza (2012), Bezerra-Gusmão (et al., 2013), Couto (et al., 2015) e Lima (et al., 2017), resultando numa baixa riqueza.

Além do que, o protocolo de coleta realizado foi diferenciado; geralmente são utilizados seis transectos (Jones \& Eggleton, 2000), neste estudo foram apenas dois, resultando em uma área total três vezes menor que o habitual. Outro ponto que pode ter interferido nos resultados são as diferenças na fitofisionomia das áreas estudadas (Alves, et al., 2011). Todavia, mesmo havendo disparidades, os resultados foram bastante semelhantes com os dados encontrados por Alves e Colaboradores (2011) para ESEC - Sérido, dentre os trabalhos vistos, o que possui maior similaridade biótica e abiótica com a Flona de Açú.

Refletindo sobre o fato do intervalo de confiança previsto pelo Sobs estar incluído nos desvios padrões sugeridos pelos estimadores utilizados, pressupõe-se um indicativo potencial de que a Flona de Açú possa ser considerada bem amostrada quanto à fauna de térmitas. Em função da curva de rarefação ter apresentado conformação sintótica e demonstrando uma tendência crescente, logo há previsão de maior número de encontros e de espécies de térmitas, a partir de um maior esforço de coleta ou maior número de transectos (Gotelli \& Colwell, 2001), além do que a curva gerada pelo Sobs, encontrava-se dentro de todos os estimadores paramétricos utilizados como comparativo, confirmando a hipótese da bem amostragem.

A diversidade encontrada para a Flona de Açú, apresentou-se pouco expressiva, com valor $H^{\prime}=1,86$ (min. 1,23 e max. 1,9). Entretanto, poucos trabalhos aferiram a diversidade de térmitas em regiões da Caatinga para serem usados como aferição. Uma das pesquisas encontradas não foi realizada em área com características semelhante, sendo esta executada em brejo de altitude no estado da Paraíba, e com um índice de diversidade maior, situado entre 2,7 e 3,2 (Lustosa Junior, 2012). Todavia, a falta de estudos, neste quesito e para este domínio morfoclimático, impossibilita uma comparação mais eficaz.

A presença abundante de vegetação rasteira e herbácea, muito comum nesta última, tem um efeito positivo no microclima, propiciando um aumento significativo em atividades de 
forrageamento próximo à superfície do solo, em detrimento da dificuldade de forrageamento durante a estação seca, época menos propensa a visualização desses insetos (Vasconcellos et al., 2007), além do que em áreas quentes e secas, colônias maiores, têm problemas com a termorregulação dos seus termiteiros (Bignell, 2011). A própria biomassa e o número de indivíduos, aparentemente, são mais altos na estação chuvosa no domínio das Caatingas (Couto et al., 2015). A redução da abundância, em parte ligada aos baixos índices de forrageamento, puderam ser refletidos nos estudos de Moura e colaboradores (2006), Mélo e Bandeira (2007) e Bezerra-Gusmão e colaboradores (2013), o que confirma o aparecimento proporcionalmente expressivo de indivíduos, mesmo com poucos transectos sendo analisados.

Ao final, foram encontradas sete espécies de térmitas, bem superior aos trabalhos realizados em uma área da Caatinga de Serra Talhada, Pernambuco, em que foram coletadas apenas duas espécies, ambas pertencente à família Termitidae (Bezerra et al., 2009); próxima aos resultados alcançados por Lima (et al., 2017), também com sete espécies, e por Santos e Gomes (2012), com nove espécies, os dois últimos autores encontraram duas famílias: Termitidae e Rhinotermitidae, com grandes semelhanças entre os gêneros encontrados, por eles e pela pesquisa realizada na Flona de Açú. Esses últimos trabalhos foram realizados na Estação Ecológica Raso da Catarina (ESEC - Raso da Catarina, Bahia) (Santos \& Gomes, 2012). Valores muitos próximos também foram associados à ESEC - Aiaiuba, Ceará, onde foi identificado oito espécies (Souza, 2012).

Outros autores, na Caatinga paraibana, como Couto (et al., 2015) relatou 45 espécies, 20 gêneros e três famílias, Vasconcellos (et al., 2010) verificou 26 espécies, 19 gêneros e três famílias, enquanto que Lustosa Junior (2012) identificou 46 morfoespécies, pertencentes a 10 gêneros e três famílias, Termitidae, Rhinotermitidae e Kalotermitidae, todos em locais serranos ou úmidos. No caso da Família Rhinotermitidae, somente o gênero Heterotermes, que também foi relatado para a pesquisa realizada na Flona de Açú foi descrito, o que reforça a ampla presença desse gênero neste domínio. Um número bem superior foi encontrado por Moura (2012), com 71 morfoespécies em brejos de altitude, pertencentes a 36 gêneros e três famílias. Todas estas pesquisas, em ambientes com condições bem diferente dos encontrados na Flona de Açu.

No estado do Rio Grande do Norte, para a ESEC - Seridó, Alves e colaboradores (2011) encontrou 11 espécies, pertencentes a três famílias, Termitidae, Kalotermitidae e Rhinotermitidae. Os autores sugerem que distúrbios antrópicos infligidos por décadas nessa área de preservação, associado a concentrações de chuvas em poucos meses e baixa produção de serapilheira, favoreceram uma redução na riqueza de térmitas. Enquanto que Souza (2012) verificou seis espécies em uma fazenda da cidade de João Câmara/RN, propondo que alguns pontos, como a presença de chuvas no período de coleta tenham aumentando a promoção de diversas espécies de insetos, e diminuído a captura de cupins pelos lagartos, uma vez que esta pesquisa a participação desses insetos na dieta de lagartos; visto a seleção por presas com maior ganho líquido energético.

Ressalta-se que, dentre os trabalhos usados como comparativos, as áreas que possuem maior similaridade abiótica e biótica com a Flona de Açú foram Bezerra (et al., 2009), Alves (et al., 2011) e Santos \& Gomes (2012), e estes apresentaram valores numericamente semelhantes, 
entretanto com resultados distintos quanto a presença/ausência de algumas espécies, embora maior parte delas sejam comuns aos trabalhos. Valores divergentes refletem a heterogeneidade da Caatinga, e suas peculiaridades quanto ao clima, geomorfologia e aspectos vegetacionais, as diferenças principais entre os brejos de altitude, regiões serranas e as áreas baixas demonstram não só aspectos abióticos, como também, que a fauna presente nestes locais dissentem um do outro, corroborando a importância de se estudar as mais diversas fisionomias da Caatinga e, as singularidades dos térmitas nestas áreas (Couto et al., 2015).

Quanto a composição das espécies coletadas a baixa frequência da família Rhinotermitidade, pode estar ligado ao fato de ser a segunda menor família de térmitas no Brasil, bem como aos seus hábitos de nidificação, costumeiramente, específicos como ninhos difusos no solo, que dificulta a possibilidade de encontrá-los no ambiente (Krishna et al., 2013). Esta família também possui uma maior distribuição em regiões de clima temperado e subtropical (Bignell, 2011), assim como a subfamília Heterotermitinae (Bignell, 2011), encontrada na Flona de Açú. O único gênero para essa subfamília, foi o Heterotermes, o qual possui 30 espécies, oito delas em regiões Neotropicais. Grande parte das espécies incluídas neste gênero é originária da América do Sul, todavia possui altas taxas de invasão em outras regiões da América Latina, como Antilhas e Caribe e devido a sua grande dispersão e invasão em outras áreas, algumas espécies são consideradas como pragas (Constantino, 1999).

Em especial as espécies Heterotermes tenuis, $\mathrm{H}$. sulcatus e $\mathrm{H}$. longiceps, geralmente são descritas em trabalhos de térmitas no Brasil (Bignell, 2011) e na Caatinga (Mélo \& Bandeira, 2007; Vasconcellos et al., 2010; Alves et al., 2011; Moura, 2012; Santos \& Gomes, 2012; Viana Junior et al., 2014; Couto et al., 2015). Nas pesquisas realizadas por Alves (et al., 2011) e Mélo e Bandeira (2007), os autores sugerem que a H. sulcatus, seja a mais importante na ESEC - Seridó e na Estação Experimental de São João do Cariri, respectivamente, no ciclo de madeira. Espécies deste gênero, podem tanto alimentar-se de madeira viva, preferencialmente, ou semidecomposta (Lima \& Costa-Leonardo, 2007).

Quanto à maior diversidade da família Termitidae, possivelmente, relaciona-se ao fato de que esta engloba o maior número de espécies de térmitas atualmente descritas, cerca de $75 \%$ e 90\% dos térmitas tropicais (Couto et al., 2015). O gênero Anoplotermes (Termitidae, Apicotermitinae) para o qual foram encontradas duas espécies distintas, apresenta sérios problemas de classificação, em razão do agrupamento de muitos integrantes da subfamília Apicotermitinae, que não se enquadram em outro gênero, neste clado, o que reflete em uma carência de estudos e revisões taxonômicas, sobretudo aos domínios das Caatingas (Constantino, 1999). Este gênero, atualmente, compõe-se de 28 espécies, dentre as quais, apenas uma não ocorre em regiões Neotropicais, destas 12 ocorrem no Brasil (KRISHNA et al., 2013).

Sobre os Nasutitermes (Termitidae, Nasutitermitinae), segundo gênero da família Termitidae encontrado, são catalogadas 250 espécies, com 71 em regiões neotropicais e 45 ocorrendo no Brasil (Krishna et al., 2013), seis espécies deste clado são consideradas pragas na América do Sul e duas dispersaram-se para ilhas do caribe (Bignell, 2011). Os resultados encontrados para esta subfamília, apenas um encontro e uma espécie (Nasutitermes sp.), se contrapõe a outros estudos publicados sobre a fauna de cupins em outros domínios morfoclimáticos brasileiros, em que pôde ser visto a predominância de encontros para esta 
subfamília, tanto em riqueza de espécies, quanto em número de encontros (Constantino, 2005; Vasconcellos et al., 2005; Vasconcellos et al., 2010; Souza et al., 2012; Santos, 2013). Além do que, uma espécie deste gênero, Nasutitermes Corniger, é uma das mais abundantes na América do Sul (Constantino, 1999; Krishna et al., 2013).

O gênero Amitermes (Termitidae, Termitinae), se perfaz como segundo maior gênero da subfamília Termitinae, sendo a primeira Microcerotermes, tido ainda como altamente cosmopolitano. Compreende 114 espécies descritas (Engel \& Krishna, 2004), destas apenas 11 ocorrem em regiões Neotropicais (Bignell, 2011) com cinco espécies ocorrendo no Brasil, com ampla distribuição no Nordeste, logo facilmente avistada, sendo uma delas endêmica da Caatinga, Amitermes nordestinus (Krishna et al., 2013). O segundo gênero descrito para Termitidae, Microcerotermes, possui 148 espécies catalogadas, com sete presente na região Neotropical e três ocorrendo no Brasil. Embora, seja o gênero com maior número de espécies registradas para esta pesquisa, é o com menor ocorrência no país (Krishna et al., 2013). Este gênero apresenta números expressivos de consumo de madeira/g diário, o que pontua sua importância no ciclo deste componente em regiões da Caatinga (Barca, 2012).

O último gênero, Termes (Termitidae, Termitinae), é natural da América do Sul, mas com fortes indicativos de dispersão para outras áreas da América Latina, como o Caribe (Bignell, 2011). Este gênero possui 24 espécies, oito em regiões neotropicais, sete com ampla distribuição no Brasil, principalmente na Amazônia, Mata Atlântica, Cerrado, Pantanal (Krishna et al., 2013) e com baixos relatos na Caatinga, sempre com pouco número de encontros (Rückamp et al., 2010; Vasconcellos et al., 2010; Alves et al., 2011; Lustosa Junior, 2012; Moura, 2012; Couto et al., 2015), sendo relatados, principalmente, as espécies Termes fatalis (Alves et al., 2011) e Termes medioculatus (Moura, 2012; Couto et al., 2015).

\subsection{Categorização das espécies encontradas e densidade de ninhos}

A presença de espécies humívoras, com valores acima de $42,7 \%(N=7)$, embora que com números de encontros intermediários 33\% ( $\mathrm{N}=12)$ e menor do que as espécies xilofágas, indica uma boa presença de serapilheira e alta participação na decomposição da matéria orgânica. No entanto, alguns estudos já vêm discutindo os efeitos sobre os grupos alimentares em áreas com distúrbios antropogênicos, sendo diferentes para biomas distintos. Por exemplo, em florestas úmidas, os humívoros são os mais vulneráveis, enquanto que na Caatinga, os térmitas xilófagos, são o grupo mais afetado, com redução severa tanto na diversidade, quanto na abundância (Vasconcellos et al., 2010). Em áreas de Cerrado, as espécies humívoras foram as mais abundantes (Carrijo et al., 2008), e esse resultado para os dois biomas citados é esperado, visto que mais de $50 \%$ dos gêneros da região tropical possuem este hábito alimentar (Noirot, 1991). Em virtude da estação chuvosa, também pôde ser observado uma quantidade significativa de serapilheira no solo da Flona de Açú, durantes os dias de coleta, o que pode ter auxiliado nestes encontros.

A porcentagem de espécies humívoras e xilófagas foram equivalentes, embora os consumidores de madeira tenham sido dominantes em número de encontros. As famílias que se encaixam nesse grupo, como Kalotermitidae e Rhinotermitidae, podem forragear este recurso 
em qualquer estágio de decomposição, excetuando as espécies que consomem este recurso extremamente decomposto e misturado ao solo (Lima \& Costa-Leonardo, 2007; Eggleton, 2011; Souza et al., 2012, Viana Junior et al., 2014). Deste modo, estas famílias seriam as que mais sofreriam com os impactos antropogênicos na Caatinga, todavia o fato da baixa presença dessas famílias para a Flona de Açú, também parece estar mais ligado aos seus hábitos de nidificação que dificultam a observação primária dessas espécies ou ainda ao tipo de vegetação presente na Flona.

Algumas espécies como a Heterotermes sp., possui um hábito de nidificação subterrâneo e enquadra-se na guilda alimentar xilófaga. Possivelmente, em dias mais quentes, bem como em áreas mais abertas, revolve a terra majoritariamente à noite, quando a temperatura encontra-se mais amena, logo isto pode ter afetado na baixa visualização de espécimes deste gênero (Vasconcellos et al., 2010). A relativa abundância das espécies Microcerotermes sp. e Heterotermes sp., que juntas representam $25 \%(N=12)$ do total de encontros e que possuem dieta essencialmente xilófaga, pressupõe a compreensão dessas como as mais importantes, entre os térmitas registrados, na ciclagem de madeira na Flona de Açú.

Quanto ao hábito de nidificação, valores equivalentes $(28,57 \%, N=7)$ de espécies que constroem seus ninhos em situações arborícolas ou subterrâneas, se assemelha com a maioria dos resultados vistos em outros trabalhos, pois para os achados na Flona de Açú os valores mais expressivos foram para espécies que constroem seus ninhos no interior de troncos, ou vivem nestes $(42,85 \%, N=7$ ), assim como observado por Alves (et al., 2011) na Esec - Seridó, Souza (et al., 2012) em fragmentos de Mata Atlântica e Moura (2012) em brejos de altitude da Caatinga Paraibana, no entanto ressalta-se que os dois últimos trabalhos ocorreram em áreas mais divergente da Flona de Açú.

Se contrapondo aos resultados de Lustosa Junior (2012), que encontrou para as 46 espécies relatadas, hábitos de nidificação arborícola, em sua maioria, o que pode estar relacionado, talvez, ao fato de que as suas pesquisas ocorreram em áreas serranas, à qual possui uma estrutura arbórea mais complexa e com maior grau de estratificação. A Couto (et al., 2015) que encontrou maiores relações com hábitos de nidificação subterrâneos para as espécies relatadas, também em ambientes mais úmidos da Caatinga e a Rückamp (et al., 2010), que encontrou maior densidade para ninhos arborícolas e epígeos.

A alta luminosidade, por consequência de uma baixa altura média das árvores da Caatinga (Amorim et al., 2016), pode influenciar na nidificação do gênero, Microcerotermes sp., por exemplo, também xilófago e arborícola, logo a presença de ninhos em locais estratégicos, fugindo da alta luminosidade sob períodos muito longo, é ponto chave para sucesso evolutivo deste gênero (Vasconcellos et al., 2010). Na Flona de Açú, os hábitos de nidificação e forrageamento foram bastante semelhantes, o que pode reforçar o potencial dos térmitas como indicadores biológicos de qualidade ambiental de hábitat na Caatinga (Viana Junior et al., 2014). Embora, só tinham sido coletados duas espécies que constroem ninhos arborícolas, o que representou $25 \%(N=12)$ dos encontros, mais três ninhos abandonados desse tipo, foram visualizados durante o período de coleta, contribuindo significativamente na participação deste clado no processo de ciclagem de nutrientes (Bezerra-Gusmão et al., 2011; Bezerra-Gusmão et al., 2013). 
Embora ninhos conspícuos sejam comuns em áreas semiáridas de savanas africanas, australianas e na própria Caatinga, chegando a 200 ninhos/ha (Bignell, 2011), esta conformação não foi encontrada para a Flona de Açú. Vasconcellos e colaboradores (2008) propõe que maiores volumes de ninhos podem estar relacionados com o grau de recuperação da cobertura da vegetação em locais que sofreram forte interferência antrópica, bem como menores oscilações dos elementos climáticos, alta disponibilidade de recurso e tamanhos das colônias. Estes fatores preponderam a importância de se estudar as assembleias de térmitas como indicadores de qualidade ambiental (Vasconcellos et al., 2008).

Verificando os trabalhos publicados que descreviam a densidade de termiteiros em áreas da Caatinga, foi visto que Alves e colaboradores (2011) constataram em sua pesquisa uma densidade relativamente baixa, cerca de 0,7 ninhos/ha, enquanto que Bezerra-Gusmão (et al., 2011) encontrou uma densidade de 76 ninhos/ha na ESEC - São João do Cariri, apenas para espécie Constrictotermes cyphergaster (Termitidae: Nasutitermitinae), em uma trabalho posterior, utilizando como base a mesma espécie e a mesma área, foi encontrado valores em torno de 272 ninhos/ha (Bezerra-Gusmão et al., 2013).

Em uma outra área de Caatinga, Lustosa Junior (2012) encontrou uma densidade média de 36 ninhos de térmitas/ha em uma área serrana da Caatinga paraibana, já Vasconcellos (et al., 2010), encontrou cerca de 89 ninhos/ha, Moura (2012), em estudos realizados em brejos de altitude inseridos no domínio morfoclimático da Caatinga, encontrou valores que giravam entre 3 a 71,8 ninhos conspícuos ativos/ha. Os valores encontrados para Flona de Açú corroboram a expressiva presença desses artrópodes na ciclagem de nutrientes na Flona de Açú, com 131,86 ninhos ativos/ha.

Compreender os comportamentos forrageadores e hábitos de nidificação dos térmitas, é observar, à longo prazo, como impactos antropogênicos podem alterar as assembleias destes insetos; as singularidades, que muitas vezes passam despercebidas. A cadência de um grupo alimentar e ascensão de outro, podem funcionar como indicativos de distúrbios significativos (Barca, 2012). Assim, refletir acerca do papel destes organismos no ciclo do carbono, nos processos de decomposição, no consumo da madeira e húmus, é elucidar-se de seu extraordinário papel na manutenção de ecossistemas (Barca, 2012), e deste ponto lutar por estratégias significativas de preservação.

\section{CONCLUSÕES}

A baixa riqueza encontrada na Flona de Açú, pode estar relacionada ao menor esforço amostral, redução no número de transecto comumente utilizados e diferenças na fitofisionomia das áreas comparadas, logo mais espécies de térmitas poderiam ser coletadas, bem como a possibilidade de um número maior de encontros. Todavia, mesmo havendo disparidades, os resultados foram bastante semelhantes com os dados encontrados por Alves e Colaboradores (2011) para ESEC - Sérido, dentre os trabalhos vistos, o que possui maior similaridade biótica e abiótica com a Flona de Açú. Ressalta-se que a pesquisa realizada pressupõe a Flona de Açú como bem amostrada quanto à fauna de térmitas, com base na visualização do intervalo de confiança 
previsto pelo Sobs e o fato deste incluir-se nos desvios padrões sugeridos em todos os estimadores utilizados neste estudo.

A família Termitidae, foi considerada dominante tanto em número de espécies, quanto em número de encontros, este fato relaciona-se a sua ampla distribuição nos ecossistemas neotropicais e sua larga presença na Caatinga. Os resultados encontrados para a subfamília Nasutitermitinae, apenas um encontro, se contrapõe a outros estudos publicados sobre a fauna de cupins nos domínios morfoclimáticos brasileiros, tanto em riqueza de espécies, quanto em número de encontros. A espécie com maior número de encontros, foi Amitermes sp., visto que este gênero possui forte presença no Nordeste e na Caatinga, o que facilita seu avistamento.

A porcentagem de espécies humívoras e xilófagas foram equivalentes, embora os consumidores de madeira tenham sido dominantes em número de encontros. A expressiva presença de espécies humívoras, indica uma boa presença de serapilheira e alta densidade de decomposição da matéria orgânica. A relativa abundância das espécies Microcerotermes sp. e Heterotermes sp., que possuem dieta essencialmente xilófagas, coaduna-as como as mais importantes, entre os térmitas registrados, na ciclagem de madeira na Flona de Açú.

Quanto aos hábitos de nidificação, os valores mais expressivos foram para espécies que constroem seus ninhos no interior de troncos, ou vivem nestes $(42,85 \%, N=7)$. 0 fato dos hábitos de nidificação e forrageamento terem sido bastante semelhantes, reforça o potencial desses artrópodes como indicadores biológicos de qualidade ambiental de hábitat na Caatinga, visto que qualquer ascensão ou diminuição destes grupos em estudos posteriores, podem indicar possíveis distúrbios. A densidade, relativamente alta, de ninhos/ha, 131,86 ninhos/ha, estimada para Flona de Açú, revela uma forte presença destes insetos nesta área de Caatinga.

\section{REFERÊNCIAS}

AB'SABER, A. N. (1974). O domínio morfoclimático das caatingas brasileiras. São Paulo: USP/IGEUG, Geomorfologia, (43).

Alves, W. D. F., Mota, A. S., De Lima, R. A. A., Bellezoni, R., \& Vasconcellos, A. (2011). Termites as bioindicators of habitat quality in the Caatinga, Brazil: is there agreement between structural habitat variables and the sampled assemblages?. Neotropical entomology, 40(1), 39-46, doi: 10.1590/S1519-566X2011000100006.

Amorim, L. D. M., de Sousa, L. D. O. F., Oliveira, F. F. M., Camacho, R. G. V., \& de Melo, J. I. M. (2016). Fabaceae na Floresta Nacional (FLONA) de Assú, semiárido potiguar, nordeste do Brasil. Rodriguésia, - Instituto de Pesquisas Jardim Botânico do Rio de Janeiro, 67(1), 105123, doi: 10.1590/2175-7860201667108.

Barca, R. R. B. (2012). Estrutura da população de Microcerotermes exiguus (Isoptera: Termitidae) e sua partuicipação no consumo de madeira em uma área de caatinga, Nordeste, Brasil (Dissertação de Mestrado). Universidade Federal do Rio Grande do Norte, Natal, RN, Brasil.

Bezerra, Y. B. S., Oliveira, C. R. F., Matos, C. H. C., Silva, M. L. L. S., \& Ferraz, C. S. (Outubro, 2009). Diversidade de cupins em áreas de caatinga em Serra Talhada - PE. IX Jornada de Ensino, 
Pesquisa e Extensão. VI Semana Nacional de Ciência e Tecnologia. UFRPE. Recife, PE, Brasil, 9.

Bezerra-Gusmão, M. A., Barbosa, J. R. C., Barbosa, M. R. D. V., Bandeira, A. G., \& Sampaio, E. V. (2011). Are nests of Constrictotermes cyphergaster (Isoptera, Termitidae) important in the $C$ cycle in the driest area of semiarid caatinga in northeast Brazil?. Applied Soil Ecology, 47(1), 1-5, doi: 10.1016/j.apsoil.2010.11.003.

Bezerra-Gusmão, M. A., Marinho, R. A., Kogiso, K. A., Bandeira, A. G., \& Barbosa, M. R. V. (2013). Nest dynamics of Constrictotermes cyphergaster (Termitidae, Nasutitermitinae) and its association with the supporting vegetation in a semiarid area, northeast, Brazil. Journal of arid environments, 91, 1-6, doi: 10.1016/j.jaridenv.2012.11.003.

BIGNELL, D. E. (2011). Morphology, Physiology, Biochemistry and Functional Design of the Termite Gut: An Evolutionary Wonderland. In BIGNELL, D. E.; ROISIN, Y.; LO, N. (Ed.), Biology of Termites: A Modern Synthesis (pp. 375-412). Dordrecht: Springer, doi: 10.1007/978-90481-3977-4.

Bignell, D. E., \& Eggleton, P. (2000). Termites in ecosystems. In Termites: evolution, sociality, symbioses, ecology (pp. 363-387). Springer: Dordrecht, doi: 10.1007/978-94-017-3223-9_17.

Carrijo, T. F., Brandão, D., de Oliveira, D. E., Costa, D. A., \& Santos, T. (2009). Effects of pasture implantation on the termite (Isoptera) fauna in the Central Brazilian Savanna (Cerrado). Journal of Insect Conservation, 13(6), 575-581, doi: doi.org/10.1007/s10841-008-9205-y.

Castelletti, C. H. M., Silva, J. M. C. D., Tabarelli, M., \& Santos, A. M. M. (2003). Quanto ainda resta da Caatinga? Uma estimativa preliminar. In Silva, J. D., Tabarelli, M., Fonseca, M. D., \& Lins, L. V. (Orgs). Biodiversidade da Caatinga: áreas e ações prioritárias para a conservação (777796). Ministério do Meio Ambiente: Brasília.

Bignell, D. E. (2011). Morphology, Physiology, Biochemistry and Functional Design of the Termite Gut: An Evolutionary Wonderland. In BIGNELL, D. E.; ROISIN, Y.; LO, N. (Ed.), Biology of Termites: A Modern Synthesis (pp. 375-412). Dordrecht: Springer, doi: 10.1007/978-90-4813977-4.

Constantino, R. (1999). Chave ilustrada para identificação dos gêneros de cupins (Insecta: Isoptera) que ocorrem no Brasil. Papéis avulsos de Zoologia, 40(25), 387-448.

Constantino, R. Padrões de diversidade e endemismo de térmitas no bioma cerrado (2005). IN: Scariot, A. O., J; Silva, C. S; Felfili, J. M. Biodiversidade, Ecologia e Conservação do Cerrado. Ministério do Meio Ambiente, Brasília, 2005.

Constantino, R. (2005). Padrões de diversidade e endemismo de térmitas no bioma Cerrado. In Scariot, A., Sousa-Silva, J. C., \& Felfili, J. M. Cerrado: ecologia, biodiversidade e conservação (319-333). Ministério do Meio Ambiente: Brasília.

Costa, C. C. A., Dantas, I. M., Camacho, R. G. V., Souza, A. M., \& Silva, N. F. Produção de Serapilheira na Caatinga da Floresta Nacional do Açú-RN. Revista Brasileira de Biociências, 
5(S1), 246-248. $\quad$ Recuperado de http://www.ufrgs.br/seerbio/ojs/index.php/rbb/article/download/273/241.

Couto, A. A., Albuquerque, A. C., Vasconcellos, A., \& Castro, C. C. (2015). Termite assemblages (Blattodea: Isoptera) in a habitat humidity gradient in the semiarid region of northeastern Brazil. Zoologia, 32(4), 281-288, doi: 10.1590/S1984-46702015000400003.

Eggleton, P. (2011). An Introduction to Termites: Biology, Taxonomy and Functional Morphology. In BIGNELL, D. E.; ROISIN, Y.; LO, N. (Ed.), Biology of Termites: A Modern Synthesis (pp. 1-26). Dordrecht: Springer, doi: 10.1007/978-90-481-3977-4.

Engel, M. S., \& Krishna, K. (2004). Family-group names for termites (Isoptera). American Museum of Natural History, 3432, 1-9, doi: 10.1206/0003-0082(2004)432<0001:FNFTI>2.0.CO;2.

Ferreira, E. V. O., Junior, V. M. A. V. I., \& do Nascimento, E. G. P. C. (2011). Ação dos térmitas no solo. Ciência Rural, 41(5), 804-811, Recuperado de http://submission.scielo.br/index.php/cr/article/download/37249/4153.

Gotelli, N. J., \& Colwell, R. K. (2001). Quantifying biodiversity: procedures and pitfalls in the measurement and comparison of species richness. Ecology letters, 4(4), 379-391.

Hammer, Ø., Harper, D. A. T., \& Ryan, P. D. (2001). PAST-Palaeontological statistics. Recuperado de https://www.uv.es/ pardomv/pe/2001_1/past/pastprog/past.

Holt, J. A; Coventry, R. J. (1990). Nutrient cycling in Australian savannas. Journal of biogeography, $17(4 / 5), 427-432$.

Howard, K. J., \& Thorne, B. L. (2010). Eusocial evolution in termites and Hymenoptera. In Bignell, D. E.; Roisin, Y.; Lo, N. (Ed.), Biology of termites: a modern synthesis (pp. 97-132). Dordrecht: Springer.

Jones, D. T., \& Eggleton, P. (2000). Sampling termite assemblages in tropical forests: testing a rapid biodiversity assessment protocol. Journal of applied Ecology, 37(1), 191-203.

Krishna, K., Grimaldi, D. A., Krishna, V., \& Engel, M. S. (2013). Treatise on the Isoptera of the world. (377). New York: American Museum of Natural History.

Lima, J. T., \& Costa-Leonardo, A. M. (2007). Recursos alimentares explorados pelos cupins (Insecta: Isoptera). Biota Neotropica, 7(2), 243-250, Recuperado de http://www.biotaneotropica.org.br/v7n2/pt/abstract?thematic-review+bn04007022007.

Lima, K. D. R. D., Camara, R., Chaer, G. M., Pereira, M. G., \& Resende, A. S. D. (2017). Soil fauna as bioindicator of recovery of degraded areas in the Caatinga biome. Revista Caatinga, 30(2), 401-411.

Lo, N., \& Eggleton, P. (2010). Termite phylogenetics and co-cladogenesis with symbionts. In Bignell, D. E.; Roisin, Y.; Lo, N. (Ed.), Biology of termites: a modern synthesis (pp. 97-132). Dordrecht: Springer. 
Lustosa Junior, N. (2012). Termitofauna em ambiente serrano no semiárido nordeste do Brasil (Relatório de Estágio Supervisionado). Universidade Estadual da Paraíba, Campina Grande, $\mathrm{PB}$, Brasil.

Maciel, B. A. (2010). Unidades de conservação no bioma Caatinga. In Gariglio, M. A., SAMPAIO, E. D. S., Cestaro, L. A., \& Kageyama, P. Y. (Orgs), Uso sustentável e conservação dos recursos florestais da Caatinga (pp. 76-81). Brasília: Ministério do Meio Ambiente.

Magurran, A. E. (2011). Medindo a diversidade biológica. Curitiba: Editora da UFPR.

Mélo, A. C. S., \& Gomes, A. G. (2007). Consumo de madeira por Heterotermes sulcatus (Isoptera: Rhinotermitidae) em ecossistema de Caatinga no Nordeste do Brasil. Oecologia Brasiliensis, 11(3), 350-355, Recuperado de https://dialnet.unirioja.es/descarga/articulo/2685171.pdf.

Menezes, V. A., Dutra, G. F., \& Rocha, C. F. (2008). Feeding habits of the endemic tropical parthenogenetic lizard Cnemidophorus nativo (Teiidae) in a restinga area of northeastern Brazil. Journal of Natural History, 42(39-40), 2575-2583, doi: 10.1080/00222930701637423.

Moura, F. M. S. (2012). Diversidade de cupins (Insecta, Isoptera) em brejos de altitude: variação espacial, influência de fatores ambientais e similaridade com domínios morfoclimáticos brasileiros (Tese de Doutorado). Universidade Federal da Paraíba, João Pessoa, PB, Brasil.

Moura, F. M. S., Vasconcellos, A., Araújo, V. F. P., \& Bandeira, A. G. (2006). Seasonality in foraging behaviour of Constrictotermes cyphergaster (Termitidae, Nasutitermitinae) in the Caatinga of northeastern Brazil. Insectes Sociaux, 53(4), 472-479, doi: 10.1007/s00040-005-0899-0.

Noirot, C. (August, 1991). From wood-to humus-feeding: an important trend in termite evolution. First European Congress of Social Insects. Leuven University Press. Leuven, Belgium.

PMA. Prefeitura Municipal de Assú (2015, Setembro). Informações Sócio-Econômicas (Site da Prefeitura). Recuperado de http://assu.rn.gov.br/.

Prado, D. E. (2003). As caatingas da América do Sul. Ecologia e conservação da Caatinga. In Leal, I. R., Tabarelli, M., \& Da Silva, J. M. C. (Ed.). Ecologia e conservação da Caatinga (3-74). Recife: Editora Universitária UFPE.

Rückamp, D., Amelung, W., Theisz, N., Bandeira, A. G., \& Martius, C. (2010). Phosphorus forms in Brazilian termite nests and soils: relevance of feeding guild and ecosystems. Geoderma, 155 (3-4), 269-279, doi: 10.1016/j.geoderma.2009.12.010.

Sampaio, E. V. S. B. (2010). Características e potencialidades. In Gariglio, M. A., SAMPAIO, E. D. S., Cestaro, L. A., \& Kageyama, P. Y. Uso sustentável e conservação dos recursos florestais da caatinga (29-48). Brasília: Ministério do Meio Ambiente.

Santos, C. A. B., \& Gomes, R. L. (2012). Caracterização dos térmitas na estação ecológica Raso da Catarina, Jeremoabo, Bahia. Revista Semiárido de Visu, 2(1), 210-218, doi: 10.1080/00222930701637423.

Santos, Q. C. L. (2013). Diversidade de Isoptera (Arthropoda, Dictyoptera) e efeito de fatores ambientais sobre sua distribuição em três áreas no município de Cláudia - MT, Amazônia 
Meridional (Dissertação de Mestrado). Universidade Federal de Mato Grosso, Cuiabá, MT, Brasil.

Souza, H. B. A. (2012). A diversidade de lagartos é mediado pelos térmitas? (Dissertação de Mestrado). Universidade Federal do Rio Grande do Norte, Natal, RN, Brasil.

Souza, H. B. D. A., Alves, W. D. F., \& Vasconcellos, A. (2012). Termite assemblages in five semideciduous Atlantic Forest fragments in the northern coastland limit of the biome. Revista Brasileira de Entomologia, 56(1), 67-72, doi: 10.1590/S0085-56262012005000013.

Rouland-Lefèvre, C. (2010). Termites as pests of agriculture. In BIGNELL, D. E.; ROISIN, Y.; LO, N. (Ed.), Biology of Termites: A Modern Synthesis (pp. 499-517). Dordrecht: Springer, doi: 10.1007/978-90-481-3977-4.

Ugland, K. I., Gray, J. S., \& Ellingsen, K. E. (2003). The species-accumulation curve and estimation of species richness. Journal of Animal Ecology, 72(5), 888-897, doi: 10.1046/j.13652656.2003.00748.x.

Vasconcellos, A., Mélo, A. C. S., Segundo, E. V., \& Bandeira, A. G. (2005). Cupins de duas florestas de restinga do nordeste brasileiro. Iheringia, Série Zoológica, 95(2), 127-131, Recuperado de http://www.scielo.br/pdf/\%0D/isz/v95n2/a03v95n2.pdf.

Vasconcellos, A., Araújo, V. F., Moura, F., \& Bandeira, A. G. (2007). Biomass and population structure of Constrictotermes cyphergaster (Silvestri)(Isoptera: Termitidae) in the dry forest of caatinga, northeastern Brazil. Neotropical Entomology, 36(5), 693-698, doi: 10.1590/S1519-566X2007000500009.

Vasconcellos, A., Bandeira, A. G., Almeida, W. O., \& Moura, F. (2008). Térmitas construtores de ninhos conspícuos em duas áreas de Mata Atlântica com diferentes níveis de perturbação antrópica. Neotropical Entomology, 37(1), 15-19, 10.1590/S1519-566X2008000100003.

Vasconcellos, A. (2010). Biomass and abundance of termites in three remnant areas of Atlantic Forest in northeastern Brazil. Revista Brasileira de Entomologia, 54(3), 455-461, doi: 10.1590/S0085-56262010000300017.

Viana-Junior, A. B., Reis, Y. T., Costa, A. P. M., \& Souza, V. B. (2014). Termite assemblages in dry tropical forests of Northeastern Brazil: Are termites bioindicators of environmental disturbances?. Sociobiology, 61(3), 324-331, doi: 10.13102/sociobiology.v61i3.324-331. 